\title{
Candida in the faeces of children receiving oral tetracycline and phenoxymethyl penicillin
}

\author{
R. J. HOLT AND R. L. NEWMAN \\ From Queen Mary's Hospital for Children, Carshalton, Surrey
}

SYNOPSIS The faeces from 100 children under 14 years receiving no antibiotic or steroid therapy were examined for Candida spp. The proportion $(8 \%)$ of positive isolates is compared with the number of Candida spp. isolated from the faeces of 50 children receiving oral tetracycline $(14 \%)$ and 59 children on phenoxymethyl penicillin $(10 \%)$ for complaints other than gastrointestinal infections. The use of selective media is described and the significance of the greatly increased numbers of Candida spp. found by these methods is discussed.

In this series no clinical manifestations of candidiasis were observed, although slightly more Candida spp. were recovered from the two groups of children on oral antibiotics than from those not on such therapy. It is suggested that, in children at least, there is a need to re-assess the significance of the presence of Candida in the bowel during the administration of oral antibiotics. The almost complete absence of active tetracycline from the faeces and the small proportion of tetracycline-sensitive bowl organisms, coupled with the low incidence of change in bowel flora in these patients, suggest that this is not the reason for proliferation of Candida in the cases that do occasionally occur.

The risk of superinfection by Candida during and after broad-spectrum antibiotic therapy was recognized soon after the introduction of antibiotics (Sharp, 1954; Weinstein, Goldfield, and Chang, 1954; Stewart, 1956; Metzger, Steigmann, Jenkins, Pamukcu, and Kaminski, 1957). Superinfection is now regarded as a commonplace phenomenon (Winner and Hurley, 1964), and it seems possible that many human beings are symptomless intestinal carriers of Candida spp. in the absence of recent antibiotic therapy. Observations on adults (Childs, 1956; Maclean, 1962) suggest that the incidence of such symptomless carriage and of clinical candidiasis may increase in patients receiving tetracycline, and as phenoxymethyl penicillin is widely used in paediatric practice it also may have some effect. No information is available, however, concerning the effect of antibiotic therapy on the bowel flora of children, and therefore we decided to investigate symptomless carriage of Candida in the bowel and to evaluate the changes in the bowel flora during oral tetracycline and penicillin therapy in children.

Faecal cultures were examined from 100 children in this hospital who had no recent history of antibiotic therapy. Children with gastro-enteritis or

Received for publication 1 September 1966. other intestinal disturbance were excluded from this series and most of the patients were newly admitted with urinary or upper respiratory infections or conditions requiring orthopaedic care.

Stools from 59 patients receiving tetracycline were also examined and from 50 patients on treatment with penicillin V. Only patients who received treatment for five days or more were included. In addition to the use of ordinary culture methods, direct smears of faeces were examined and highly selective media were used in order to assess the weight of infection with Candida when it occurred.

\section{METHODS}

Direct film preparations were examined from all clinical material under investigation. Dry films were stained by Gram, and saline-suspended wet films were examined by interference microscopy. The approximate number $\underset{\gamma}{\sigma}$ and shape of Candida spores were noted, and hyphal $\theta$ outgrowth, hyphal fragments, and active budding were $\frac{\bar{D}}{\mathbb{D}}$ sought. Dead Candida cells could be distinguished by loss of ability to retain Gram stain and by the changes of internal appearance under the interference microscope.

Faeces and mouth washings were heavily inoculated on to plates containing modified Czapek-Dox agar (Oxoid), a variation of the Czapek formula of Dox $\&$ (1910). When first prepared it has a $p H$ near 7.0, at which $\underset{\gamma}{\sigma}$ 
it is an excellent substrate for chlamydospore production by Candida albicans (Dawson, 1962). In primary faecal cultures, however, Candida is very likely to be overgrown by Enterobacteriacae, particularly Klebsiella, but this can be avoided by adjusting the $p \mathrm{H}$ to $5 \cdot 5$, together with the addition of benzylpenicillin and streptomycin, each in final concentrations of $100 \mu \mathrm{g} . / \mathrm{ml}$. Chloramphenicol, also at $100 \mu \mathrm{g} . / \mathrm{ml}$., is equally effective. This medium is sufficiently selective to allow almost pure growth of Candida after 48 hours' incubation at $28^{\circ} \mathrm{C}$.

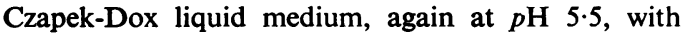
benzylpenicillin and streptomycin, was used as an enrichment medium. A heavy inoculum of clinical material was added and incubated for 24 hours at $28^{\circ} \mathrm{C}$. If, meanwhile, no growth of Candida was found on the primary plate, about $0 \cdot 1 \mathrm{ml}$. of the liquid medium was

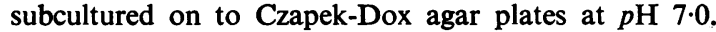
The inoculum was inserted through the medium and spread beneath the agar layer in the method reported by Dawson (1962). Subcultures of primary growth were similarly inoculated and all plates examined after 24 and 48 hours' incubation at $28^{\circ} \mathrm{C}$. for chlamydospore formation: chlamydospore production was much more pronounced at $p \mathrm{H} \mathrm{7.0} \mathrm{than} \mathrm{at} p \mathrm{H} \mathrm{5 \cdot 5.}$

Faeces were cultured on Oxoid MacConkey agar and on simple nutrient agar plates, and faecal flora were assessed semi-quantitatively. Mouth washings and saliva were also inoculated on to blood agar plates.

All isolates of Candida were tested for germ tube production in normal fresh human serum (Taschdjian, Burchall, and Kozinn, 1960) and all those strains able to produce these extrusions and also chlamydospores were classified as Candida albicans. Attempts were made to classify further the strains not reacting in these ways on the basis of colonial characteristics and ability to ferment carbohydrates (Martin, Jones, Yao, and Lee, 1937). Since the results proved equivocal, a number of strains failing to correspond with reported species, these strains were collectively labelled 'non-albicans'.

Sensitivity to antifungal drugs was tested by the disc method against light inocula poured as even lawns over nutrient agar plates. Drug concentrations were $100 \mu \mathrm{g}$. per disc for nystatin, $50 \mu \mathrm{g}$. per disc for amphotericin B and pimaricin (natamycin).

\section{RESULTS}

The overall results are set out in Table I.

CHILDREN NOT RECEIVING ANTIBIOTICS Faeces fro.n 100 patients were examined; their ages ranged from 3 months to 13 years. All had just been admitted to this hospital, generally from home.

By direct methods Candida was found in eight.

Smear positive, culture positive .. 4

Smear positive, culture negative .. 1

Smear negative, culture positive .. 3

Two of the seven strains isolated by direct culture were classified as $C$. albicans; the remaining five failed to form germ tubes or chlamydospores. No hyphal extrusions were seen in smears. Twenty-two of the specimens of faeces from which no Candida was found by direct methods were further cultured through enrichment broth.

$\begin{array}{lll}\text { No Candida found } & \ldots & 12 \\ \text { Scanty growth of Candida } & \ldots & 3 \\ \text { Moderate growth of Candida } & \ldots & 5 \\ \text { Heavy growth of Candida } & \ldots & 2\end{array}$

All 17 isolates of Candida were sensitive to nystatin, amphotericin, and pimaricin.

CHILDREN RECEIVING ORAL ANTIBIOTICS At least three faecal specimens were examined from each child. The first was taken either immediately before therapy or on the first day of the course and an intermediate specimen was cultured on the third or fourth day, followed by a final culture two or three days after completion of the course. This lasted in most cases either five or seven days; some lasted as long as 14 days.

Oral tetracycline In most cases the dosage was in the region of $20 \mathrm{mg} . / \mathrm{kg}$. body weight per day. Most of the 50 children examined were treated for respiratory complaints.

Direct culture showed that candida was present in the initial specimen in three.

Smear positive, culture positive .. 2

Smear negative, culture positive .. 1

Two of these initial positives became negative in intermediate and final specimens.

Candida was found in six of 50 others in intermediate and final cultures (direct).

Smear positive, culture positive .. 5

Smear negative, culture positive .. 1

One became culturally positive for Candida only in the final specimen, which was smear negative. No smear showed hyphal extrusions.

When intermediate and final cultures were made on non-inhibitory media, in six out of 50 there was marked diminution or absence of their initial flora; this change in flora was in no way related to the recovery of Candida from particular patients. None of the children showed clinical symptoms during or after therapy which could be attributed to the administration of oral tetracycline.

Three of the seven strains of Candida found following therapy were identified as $C$. albicans. All seven strains were fully sensitive to the three antifungal drugs.

Oral phenoxymethyl penicillin Here again respiratory complaints were the main reason for therapy. Dosage was usually $20 \mathrm{mg}$. $/ \mathrm{kg}$. body weight per day. Specimens from 59 children were examined. 
TABLE I

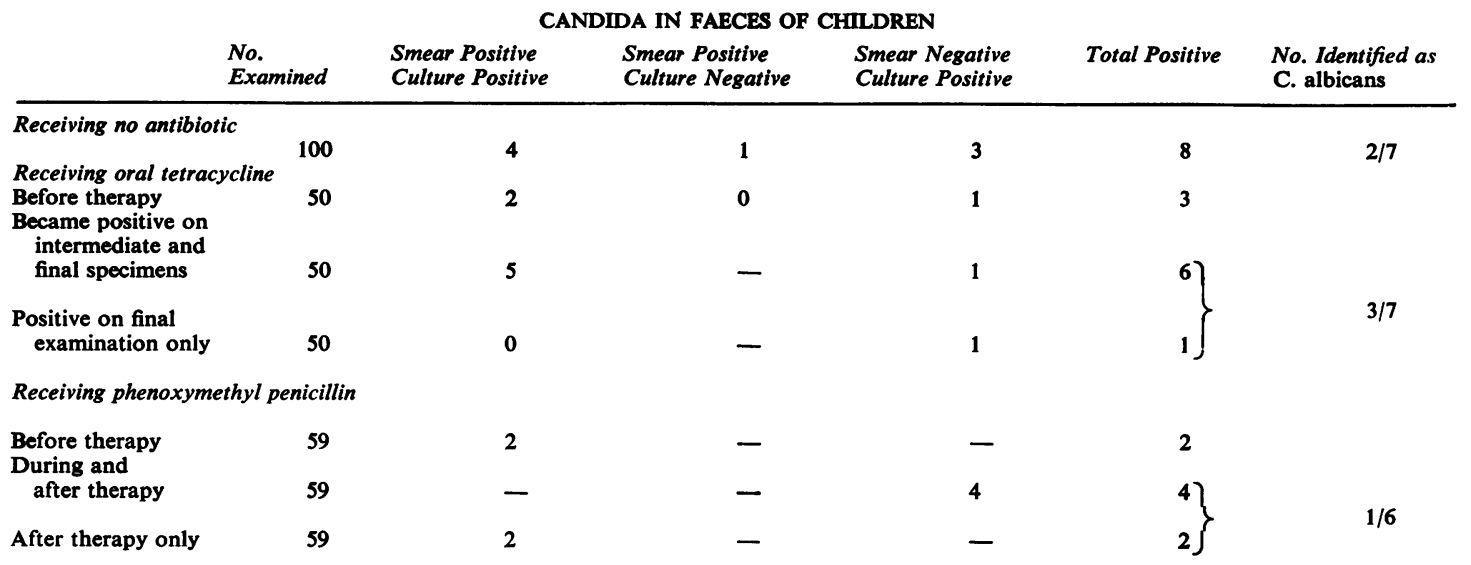

Two specimens gave direct growth of Candida before treatment. Both were smear positive. Cultures from one of these children became negative during and after therapy. Four became positive on intermediate and final direct culture; all four were smear negative. Two were culturally positive only on posttherapy examination; both were smear positive.

One of these six strains of Candida was classified as $C$. albicans; all strains were fully sensitive to antifungal drugs. No obvious changes in general faecal flora were noted.

One child had slight nausea towards the end of therapy; it was doubtful whether this was, in fact, attributable to the treatment.

MOUTH CULTURES Mouth washings or extensive swabbings from the mouths of 10 children were cultured for Candida by both direct and enrichment methods. These children had not had any recent antibiotic and were selected at random. As much material as could reasonably be obtained was used. There were usually considerable volumes of saliva present.

Direct culture showed the presence of Candida spp. in two children (both smear negative); enrichment methods gave positive findings in seven (also all smear negative). One of the Candida strains isolated was identified as $C$. albicans.

SENSITIVITY OF SPECIES OTHER THAN CANDIDA PRESENT IN THE FAECES The sensitivity of normal gut flora to tetracycline and to phenoxymethyl penicillin was tested against 10 and $50 \mu \mathrm{g}$. discs on conventional surface-seeded agar plates (Table II).

\section{DISCUSSION}

The frequency with which Candida is found in the faeces of children appears to bear a direct relation-
TABLE II

SENSITIVITY OF SPECIES OTHER THAN CANDIDA $\begin{array}{lll}\text { No. } & \begin{array}{l}\text { Sensitive to } \\ \text { Tested }\end{array} & \begin{array}{l}\text { Sensitive to } \\ \text { Phenoxymethyl } \\ \text { Penicillin }\end{array}\end{array}$

\begin{tabular}{lccc}
\hline Coliforms/aerogenes & 50 & $22 / 50$ & $0 / 50$ \\
$\begin{array}{l}\text { Proteus spp. (unclassi- } \\
\text { fied, mainly mirabilis) }\end{array}$ & 29 & $1 / 29$ & $9 / 29$ \\
Enterococci & 17 & $8 / 17$ & $5 / 17$
\end{tabular}

ship to the efficiency of the cultural methods in use. Thus the $8 \%$ faecal Candida recovery rate by direct cultural means from children not receiving oral antibiotics was increased to a figure approaching $50 \%$ by more selective methods. Similarly, in a small series of cultures of mouth secretions, two of 10 were positive by direct means but reached seven when enrichment culture was employed. The main point to emerge here is the need to scale down the scope of cultural methods to a level where positive findings indicate the presence, or the imminent risk, of candidiasis. It may well be that direct smear examinations are sufficiently precise for this purpose; certainly this method has the added advantage of speed and permits detection of dead Candida cells, while the finding of hyphal extrusions might suggest active proliferation in the lower intestine.

Candida was recovered by direct culture from $14 \%$ of children receiving oral tetracycline and from 10\% of children receiving phenoxymethyl penicillin but only six of the 20 Candida strains isolated were identified as Candida albicans, It is difficult to assess the significance of this finding in view of the fact that many clinicians view the presence in the gut of any member of the genus with concern. It should be noted that in this investigation no special steps were taken to exclude the risk of perianal contamination. 
Smits, Prior, and Arblaster (1966) suggest special techniques to avoid this, although they quote no figures for the likelihood of contamination of the anal margin or skin.

It is not clear precisely how a heavy, even overwhelming, proliferation of Candida in the gut can occur, often associated with systemic spread. A number of theories have been proposed: the elimination of competing organisms in the local flora, the elimination of organisms able to synthesize vitamins, and the consequent lowered host resistance due to avitaminosis, have been suggested (Conant, Smith, Baker, Callaway, and Martin, 1954). The growth of Candida in vivo may be stimulated by the antibiotic, although experiments here in vitro have failed to demonstrate this.

The high proportion of positive Candida cultures from the mouth obtained on enrichment indicates that a continual low grade commensal colonization of the buccal cavity is maintained, possibly from ingestion of Candida on foods; Candida spp., for example, may be readily isolated from the surface of fruits as well as from soil (Smith, 1954). This reservoir could promote active colonization of the gut with Candida after the disturbance or diminution of normal bowel flora following the administration of broad-spectrum chemotherapeutics. Such an alteration would necessitate both the presence of antibiotic in the faeces as a result of incomplete absorption and the sensitivity of much of the initial gut flora to that antibiotic. It is therefore of interest that assays on faeces from some of the children receiving oral tetracycline revealed very little active tetracycline, usually under $10 \mu \mathrm{g} . / \mathrm{g}$. wet faeces. Little information is available about the pharmacology of tetracycline in children, but this finding may mean that tetracycline is almost wholly absorbed in young patients. Certainly random urine samples showed extremely high tetracycline levels in these patients, in the order of 1,000 to $2,000 \mu \mathrm{g} . / \mathrm{ml}$. at about two hours after the previous oral dose. The results shown in Table II indicate that less than half of the faecal organisms examined were sensitive to tetracycline and even fewer to phenoxymethyl penicillin. However, in the present study only $12 \%$ of children showed disturbance of gut flora under the suppressive activity of oral tetracycline, and this was apparently unrelated to the presence or proliferation of Candida; no changes were noted as a result of phenoxymethyl penicillin therapy. Moreover it must be stressed that none of the patients showed the slightest sign of clinical candidiasis, a condition which is extremely uncommon in this hospital. The absence in this series of even mild gastro-intestinal symptoms associated with the proliferation of Candida in the gut corresponds with the recent findings of Smits et al. (1966), although their investigations were conducted on adult patients.

We wish to thank Professor G. T. Stewart and Dr. Brian Cromie. It is a pleasure to record the cooperation of the nursing and medical staff who were involved in this study.

\section{REFERENCES}

Conant, N. F., Smith, D. T., Baker, R. D., Callaway, J. L., and Martin, D. S. (1954). Manual of Clinical Mycology, 2nd. ed., p. 180. Saunders, Philadelphia and London.

Childs, A. J. (1956). Brit. med. J., 1, 660.

Dawson, C. O. (1962). Sabouraudia, 1, 214.

Dox, H. (1910). Fungal Media. U.S. Dept. Agn. Bur. Anim. Ind. Bull, 120,70 .

Maclean, K. (1962). Medical Treatment, 2nd. ed., p. 715. Churchill, London.

Martin, D. S., Jones, C. P., Yao, K. F., and Lee, L. E., Jr. (1937) J. Bact., 34, 99.

Metzger, W. I., Steigmann, F., Jenkins, C. J., Jr., Pamukcu, S. F., and Kaminski, L. (1957). Antibiot. Ann., 1956-57, 208.

Sharp, J. L. (1954). Lancet, 1, 390.

Smith, G. (1954). An Iniroduction to Ir.dustrial Mycology, 4th. ed., p.75. Arnold, London.

Smits, B. J., Prior, A. P., and Arblaster, P. G. (1966). Brit. med. J., $1,208$.

Stewart, G. T. (1956). Ibid., 1, 658.

Taschdjian, C. L., Burchall, J. J., and Kozinn, P. J., (1960). Amer. J. Dis. Child., 99, 212.

Weinstein, L., Goldfield, M., and Chang, T. W. (1954). New Engl. J. Med., $251,247$.

Winner, H. I. and Hurley, R. (1964). Candida Albicans. Churchill, London. 